\title{
Preparation of Nitrogen-Doped Carbon Materials from Monosodium Glutamate and Application in Reduction of p-Nitrophenol
}

\author{
Ke Ying Cai*, Ying Mei Zhou, Peng Wang, Huan Li, Yan Li, Wei Tao \\ School of Chemistry and Chemical Engineering, Xuzhou University of Technology, Xuzhou, \\ Jiangsu 221018, China
}

Received: 28th July 2017; Revised: 1st September 2017; Accepted: $7^{\text {th }}$ September 2017

Available online: 22 $2^{\text {nd }}$ January 2018; Published regularly: $2^{\text {nd }}$ April 2018

\begin{abstract}
Nitrogen-doped carbons (NCs) as supports for metal catalysts used in heterogeneous reactions are increasingly being investigated. In this work, $\mathrm{NCs}$ were prepared from monosodium glutamate (MSG) by direct carbonization, which were used as supporters to prepare $\mathrm{Bi} / \mathrm{NC}$ catalysts. The $\mathrm{Bi} / \mathrm{NC}$ catalysts were characterized by X-ray powder diffraction (XRD), X-ray photoelectron spectroscopy (XPS), transmission electron microscope (TEM), and nitrogen adsorption isotherm. The results indicate that nitrogen was doped in the formation of pyridinic $\mathrm{N}$, pyrrolic $\mathrm{N}$, and graphitic $\mathrm{N}$. The NCs possess high surface area $\left(\sim 652 \mathrm{~m}^{2} / \mathrm{g}\right)$ and uniform mesopore size $(\sim 2.11 \mathrm{~nm})$. Bismuth nanoparticles (NPs) dispersed uniformly in $\mathrm{NC}$ with diameter of $10-20 \mathrm{~nm}$. The catalytic performances were investigated using the reduction of 4-nitrophenol (4-NP) with excess potassium borohydride as a model reaction, the results indicating that the $\mathrm{Bi} / \mathrm{NC}$ catalysts have higher activity and better reusability than the $\mathrm{Bi} / \mathrm{AC}$ catalyst. Under the following conditions: $100 \mathrm{~mL}$ of $4-\mathrm{NP}(2 \mathrm{mM}), 0.03 \mathrm{~g}$ of $3 \% \mathrm{Bi} / \mathrm{NC}, n\left(\mathrm{KBH}_{4}\right): n(4-$ $\mathrm{NP})=40: 1$, and at room temperature, the rate constant $k$ can reach $0.31 \mathrm{~min}^{-1}$. Copyright (C) 2018 BCREC Group. All rights reserved
\end{abstract}

Keywords: Reduction; 4-Nitrophenol; Nitrogen-doped carbon; Monosodium glutamate

How to Cite: Cai, K.Y., Zhou, Y.M., Wang, P., Li, H., Li, Y., Tao, W. (2018). Preparation of NitrogenDoped Carbon Materials from Monosodium Glutamate and Application in Reduction of $p$-Nitrophenol. Bulletin of Chemical Reaction Engineering \& Catalysis, 13 (1): 89-96 (doi:10.9767/bcrec.13.1.1428.8996)

Permalink/DOI: https://doi.org/10.9767/bcrec.13.1.1428.89-96

\section{Introduction}

Carbon materials have attracted more and more attention owing to their broad availability, environmental acceptability, corrosion resistance, and unique surface properties [1]. All these aspects make carbon materials promising

* Corresponding Author.

E-mail: caikeying@163.com (Cai, K.Y.)

Telp.: +86-13852081673; Fax.: +86-516-85608300 for various application, including energy conversion and storage, electrocatalysis, heterogeneous catalysis, and sensors. Other than morphology control, chemical doping is another important approach to modify the property of carbon materials, which has been demonstrated as a successful method to tune carbon's electronic structure, increasing active sites and enhancing catalytic activity significantly [2]. Of all the alternative dopant elements, the nitrogeninvolved modification has attracted much atten- 
tion because the charge distribution of carbon atoms will be influenced by the neighbor nitrogen atoms [3]. Thus nitrogen-doped carbon (NC) has different properties compared with the pristine carbon, which broaden its applications [4]. For instance, NC activated by doped nitrogen can be used as a catalyst in oxygen reduction reaction (ORR) $[5,6]$ and heterogeneous catalytic reactions [2]. NC can also be used as a catalyst carrier in some heterogeneous catalytic reactions, which can anchor the metal NPs firmly [7]. Moreover, NCs can be used in batteries, sensors, and ultracapacitors [4].

NC can be obtained through two different ways: direct synthesis and post treatment. Direct synthesis includes chemical vapor deposition (CVD), segregation growth, solvothermal, and arc-discharge approaches. Post treatment includes thermal treatment, plasma treatment, and $\mathrm{N}_{2} \mathrm{H}_{4}$ treatment [1]. The main shortcoming of post treatment is that it entails numerous synthetic steps, hence direct synthesis is more commonly utilized in the synthesis of NC [8]. Furthermore, nitrogen distribution can be more uniform in carbon material by direction synthesis. Many substances can be used as precursors for $\mathrm{NC}$ materials by direct synthesis such as gelatin [9], chitosan [10], pyrrole [11], dopamine [12], and aniline [13].

Monosodium glutamate (MSG) with amino group is a kind of perfect raw material for $\mathrm{NC}$, because it is inexpensive and easy to obtain. To the best of our knowledge, few NC materials from MSG were studied. Yan and co-workers synthesized porous carbon nanoflakes by cooking a mixture of MSG and sodium chloride, and the material can facilitate persistent and fast ion transport and shorten diffusion pathways for high-performance supercapacitor applications [14]. But there is no report on NC from MSG used as a metal catalyst support.

In this work, NC was synthesized by pyrolysis of MSG which was employed as a source of carbon and nitrogen. Then the as-synthesized $\mathrm{NC}$ was used as a support to prepare $\mathrm{Bi} / \mathrm{NC}$ catalyst. The catalytic performances of $\mathrm{Bi} / \mathrm{NC}$ catalyst were investigated using the reduction of $p$-nitrophenol (4-NP) to $p$-aminophenol (4$\mathrm{AP})$ as a model reaction under mild conditions (Scheme 1). The 4-NP threatens seriously human health and natural environment due to its structural stability, difficult degradation, and water solubility, which are from the production of pesticides, herbicides, insecticides and synthetic dyes cause serious pollution [15]. But the reduced product 4-AP is less poisonous and more easily degradable than 4 -NP. It is therefore necessary to develop sustainable and active catalyst for the reduction of 4-NP. Nobel metals, such as: Pd [16], Au [17], Ag [18], etc., are often used as active components in the catalysts for the reduction.

Compared with noble metals, bismuth is cheap and easily fabricated by reduction with reducing agent such as sodium borohydride and hydrazine hydrate. More importantly, bismuth is a kind of environment-friendly element. Bismuth (III) salts are considered safe and non-toxic probably due to their insolubility in neutral aqueous solutions [19]. Therefore, bismuth and its salts appeared as suitable regents for design of "eco-friendly catalyst" for the reduction of $4-\mathrm{NP}$.

\section{Materials and Methods}

\subsection{Materials}

Bismuth nitrate pentahydrate, MSG, potassium hydroxide, and 4-NP were purchased from Tianjin Fuchen Reagent Co. Ltd. (China). Potassium borohydride (95\%) was purchased from Sinopharm Chemical Reagent Co. Ltd. (China). All reagents were of analytic grade and used without further purification. Ultrapure water was used throughout the whole experiment.

\subsection{Catalyst preparation}

NC was synthesized by pyrolysis of MSG. Typically, $5.0 \mathrm{~g}$ of MSG and $1.0 \mathrm{~g}$ of $\mathrm{KOH}$ were carbonized in a horizontal tube furnace at a

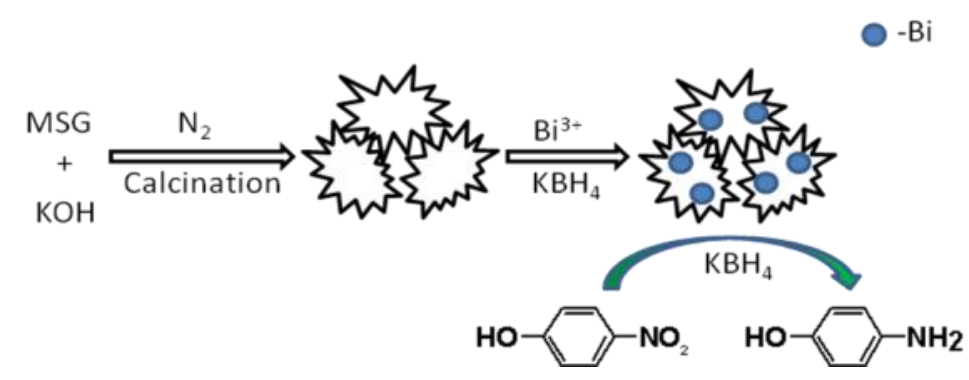

Scheme 1. Schematic illustration of the synthesis process of the Bi/NC 
rate of $3{ }^{\circ} \mathrm{C} \cdot \mathrm{min}^{-1}$ under nitrogen flow at $700{ }^{\circ} \mathrm{C}$ for $2 \mathrm{~h}$ and then cooled to room temperature spontaneously. The obtained substance was washed two times with $\mathrm{HCl}(10 \mathrm{wt} \%)$ to remove inorganic salts, thoroughly washed with abundant water, and then dried at $100{ }^{\circ} \mathrm{C}$ for 2 $\mathrm{h}$, which was denoted as NC. Bismuth nitrate pentahydrate $(0.07 \mathrm{~g})$ was dissolved in $5 \mathrm{~mL}$ of dilute nitric acid at room temperature. After bismuth nitrate dissolved completely, $15 \mathrm{~mL}$ of water was added and stirred. Subsequently, $1 \mathrm{~g}$ of $\mathrm{NC}$ was added to the solution and sonicated for $30 \mathrm{~min}$ to disperse them. Following that, 10 $\mathrm{mL}$ of potassium borohydride $(0.5 \mathrm{M})$ solution was dropped into the mixture and stirred constantly for $1 \mathrm{~h}$.

The mixture was filtered, and the obtained solids were washed with water to neutral and dried at $80{ }^{\circ} \mathrm{C}$ for $4 \mathrm{~h}$. In the composite, the $\mathrm{Bi}$ content was about $3 \%$, hence the as-prepared composite denoted as $3 \% \mathrm{Bi} / \mathrm{NC}$. The composites with different $\mathrm{Bi}$ content, such as $1 \% \mathrm{Bi} / \mathrm{NC}$ and $5 \% \mathrm{Bi} / \mathrm{NC}$ were prepared using the same method. For the purpose of comparison, activated carbon (AC) as a carrier, the $3 \% \mathrm{Bi} / \mathrm{AC}$ was also prepared using the same method.

\subsection{Catalyst characterization}

The X-ray diffraction (XRD) patterns were recorded on a diffractometer (Rigaku Ultima IV) equipped with a $\mathrm{Cu}-\mathrm{K}_{\alpha}$ radiation source. It was operated under the following conditions: tube voltage $40 \mathrm{kV}$, current $40 \mathrm{~mA}, \lambda=0.15406$ $\mathrm{nm}$, and scanning range $2 \theta=10-70^{\circ}$. The morphology and microstructure of the as-prepared samples were investigated with transmission electron microscope (TEM.JEOL JEM-2100). Xray photoelectron spectroscopy (XPS) was obtained by X-ray photoelectron spectrometer (Thermo ESCALAB 250XI) using Al-Ka X-ray as the excitation source $(225 \mathrm{~W})$. The specific surface area of the carbons were determined in a Micromeritics sorptometer at $77 \mathrm{~K}$. Before analysis, the samples were evacuated for $12 \mathrm{~h}$ at $250{ }^{\circ} \mathrm{C}$ under vacuum. The surface areas were calculated using the Brunauer-EmmettTeller (BET) method based on adsorption data in the relative pressure of $0.01-0.2$, and their pore diameter distributions were calculated using the Barrett-Joyner-Halenda (BJH) method.

\subsection{Catalytic reduction of 4-NP}

The reduction of $4-\mathrm{NP}$ catalyzed by $\mathrm{Bi} / \mathrm{NC}$ catalyst in the presence of an excess amount of potassium hydroxide was studied, monitored with a UV-Vis spectroscopy to examine the catalytic activity. In a typical reaction, $5 \mathrm{~mL}$ of 4-NP $(40 \mathrm{mM})$ and $75 \mathrm{~mL}$ of water were mixed with $0.03 \mathrm{~g}$ of $\mathrm{Bi} / \mathrm{CN}$ particles in a beaker at room temperature. A fresh prepared aqueous solution of $20 \mathrm{~mL} \mathrm{KBH} 4(0.4 \mathrm{M})$ was then added with constant stirring. During the reduction, the yellow color of solution gradually vanished, indicating the reduction of 4-NP. Samples were directly withdrawn from the reaction mixture at certain time intervals followed by measuring UV-Vis absorption at 400 $\mathrm{nm}$. The rate constant of the reaction was determined by measuring the absorbance change in intensity at $400 \mathrm{~nm}$ with time.

To study the reusability of the as-prepared catalysts, the used $\mathrm{Bi} / \mathrm{NC}$ catalysts were separated from the reaction solution by centrifugation after the reduction. The recycled particles were washed with ethanol and water 3 times respectively and reused. Since the absorbance is proportional to the concentration of $4-\mathrm{NP}$, the conversions were also determined by the absorbance changes of reaction solution at 400 $\mathrm{nm}[17,18]$.

\section{Results and Discussion}

\subsection{Characterization of the catalysts}

The phase structure of the sample was obtained through XRD measurement. As shown in Figure 1, the broad peaks located around $25^{\circ}$ for all $\mathrm{Bi} / \mathrm{NC}$ and $\mathrm{Bi} / \mathrm{AC}$ samples can be index as (002) diffraction planes of the hexagonal phase graphite (JCPDS No. 41-1487) [20]. The broad pattern of diffraction peaks indicates the poor graphitization degree for all products [21]. Three diffraction peaks at approximately $27.0^{\circ}$, $37.8^{\circ}$, and $39.5^{\circ}$ in the XRD patterns of

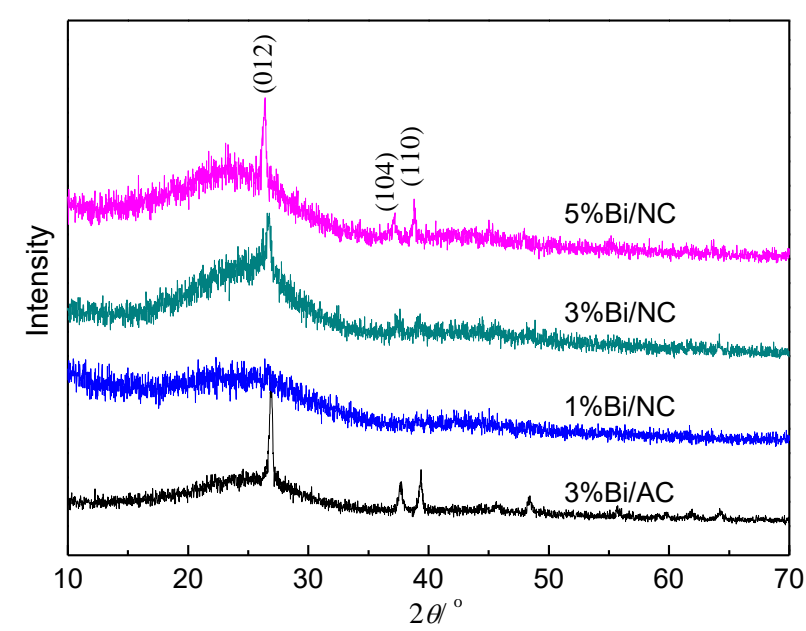

Figure 1. X-ray diffraction (XRD) patterns of the catalysts 
$3 \% \mathrm{Bi} / \mathrm{NC}, 5 \% \mathrm{Bi} / \mathrm{NC}$, and $3 \% \mathrm{Bi} / \mathrm{AC}$, can be indexed to (012), (104) and (110) planes of metallic bismuth (JCPDS No. 05-0519), respectively [22]. However, there is no the three corresponding peaks in the XRD pattern of $1 \% \mathrm{Bi} / \mathrm{NC}$ because of low bismuth content in it.

To determine the catalyst elemental content and the states of the $\mathrm{N}$ atoms, XPS was employed. As shown in Figure 2a, the XPS spectra reveal the presence of $\mathrm{C}, \mathrm{N}, \mathrm{O}$, and Bi elements in $3 \% \mathrm{Bi} / \mathrm{NC}$, but there is no $\mathrm{N}$ element in $3 \% \mathrm{Bi} / \mathrm{AC}$. The results demonstrated that nitrogen had been successfully doped into the NC's lattice. The relative surface concentrations of $\mathrm{C}, \mathrm{N}, \mathrm{O}$, and Bi calculated from XPS analysis were $67.86,4.87,27.04$, and 0.23 at $\%$ in $3 \% \mathrm{Bi} / \mathrm{NC}$, respectively. The $\mathrm{N} 1 \mathrm{~s}$ spectrum (Figure 2b) can be fitted into three peaks, which can correspond to pyridinic N $(398.1 \mathrm{eV})$, pyrrolic N (399.3 eV), and graphitic N (400.5 $\mathrm{eV})[4,23]$. The $\mathrm{N}$ atom percent distribution in pyridinic $\mathrm{N}$, pyrrolic $\mathrm{N}$, and graphitic $\mathrm{N}$ was $30.7,25.3$, and 44.0 at \%, respectively. This result indicates that the graphitic $\mathrm{N}$ is the main $\mathrm{N}$-style in the NC catalyst. The Bi $4 \mathrm{f}$ spectra of the as-synthesized sampled are shown in Figure 2c. The $\mathrm{Bi} 4 \mathrm{f}^{7 / 2}$ and $4 \mathrm{f}^{5 / 2}$ peaks at approximately $160.0 \mathrm{eV}$ and $165.3 \mathrm{eV}$, respectively, are considered the high-spin $\mathrm{Bi}^{3+}$ cation, which elucidates that bismuth oxide is the predominant bismuth phase in the targeted samples due to oxidation of bismuth particles in the air.

The structure and morphologies of $3 \% \mathrm{Bi} / \mathrm{NC}$ and $3 \% \mathrm{Bi} / \mathrm{AC}$ catalysts can be observed in the TEM images (Figure 3). The Bi particles distribute uniformly on the external surface of $\mathrm{NC}$ with the diameter of 10-20 nm, but the Bi particles distribute unevenly on AC with broad size distribution. The possibly reason for the result is that the $\mathrm{Bi}$ particles were strongly attracted by pyridinic $\mathrm{N}$ atoms or the carbon atoms neighboring graphitic $\mathrm{N}$ in $\mathrm{NC}[8,23]$, which can fixed $\mathrm{Bi}$ particles and impede them aggregation. However, there is no strong adsorption between $\mathrm{Bi}$ particles and $\mathrm{AC}$.

The porous structure of the as-prepared materials was measured using $\mathrm{N}_{2}$ adsorption- desorption isotherms. Figure 4 a shows a type IV isotherm for $3 \% \mathrm{Bi} / \mathrm{NC}$, indicating the existence of mesopores. The isotherm for $3 \% \mathrm{Bi} / \mathrm{AC}$ in Figure $4 \mathrm{a}$ exhibit a fairly sharp knee in the low relative pressure $\left(p / p_{0}<0.1\right)$, which is similar to the type I curve of the microporosity characteristic. The BET surface areas of $3 \% \mathrm{Bi} / \mathrm{NC}$ and $3 \% \mathrm{Bi} / \mathrm{AC}$ are $652 \mathrm{~m}^{2} / \mathrm{g}$ and $811 \mathrm{~m}^{2} / \mathrm{g}$, respectively. The pore size distribution curves were calculated by using the BJH method and are shown in Figure 4b. Most of the pores in $3 \% \mathrm{Bi} / \mathrm{NC}$ are mesopores, and the average pore diameter is about $2.11 \mathrm{~nm}$. However, in $3 \% \mathrm{Bi} / \mathrm{AC}$ most of the pores are micropores, and the average pore diameter is less than $2.0 \mathrm{~nm}$.

\subsection{Catalytic activity}

To investigate the catalytic activity of the
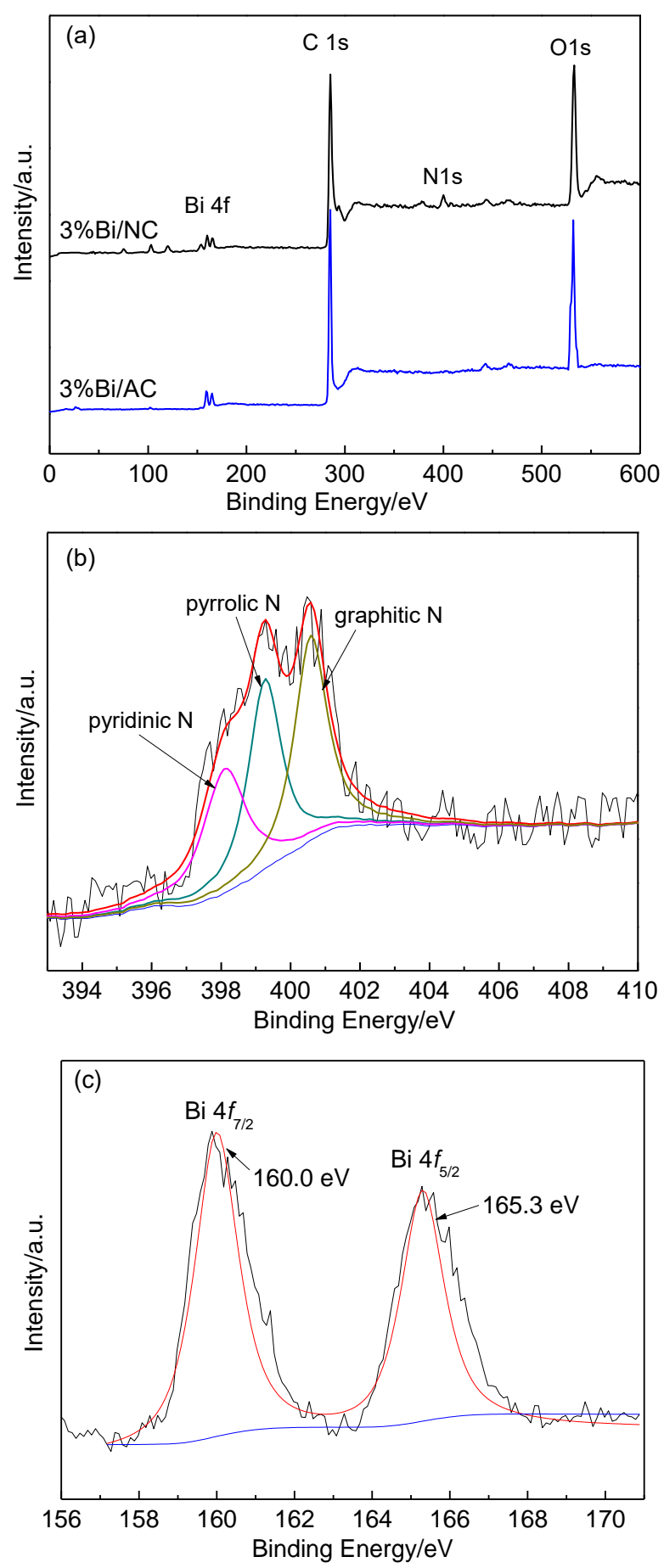

Figure 2. XPS surveys of $3 \% \mathrm{Bi} / \mathrm{NC}$ and $3 \% \mathrm{Bi} / \mathrm{AC}$ (a), high-resolution N 1s XPS spectra (b), and high-resolution Bi 4f XPS spectra (b) 
$\mathrm{Bi} / \mathrm{NC}$ and $\mathrm{Bi} / \mathrm{AC}$ catalysts, the reduction of 4 $\mathrm{NP}$ to 4-AP in the presence of excess $\mathrm{KBH}_{4}$ was chosen as a model reaction. The reaction is particularly easy to detect because there is only one product, 4-AP, and the extent of reaction can be followed by measuring the absorbance change at $400 \mathrm{~nm}$ and $300 \mathrm{~nm}$ in UV-vis. As the concentration of $\mathrm{KBH}_{4}$ largely exceeds the concentration of $4-\mathrm{NP}$, it can be considered as a constant during the reaction, and therefore the reduction can be regarded as pseudo-first-order reaction. Since the absorbance of 4 -NP is proportional to its concentration in the medium, the ratio of $A_{\mathrm{t}}(t=\mathrm{t})$ to $A_{0}(t=0)$ must be equal to the concentration ratio $C_{t} / C_{0}$ of $4-\mathrm{NP}$ [24]. The kinetic equation for the reduction can be written as Equation (1).

$$
\ln \left(A_{t} / A_{0}\right)=\ln \left(C_{t} / C_{0}\right)=-k t
$$

In this equation $k$ is the apparent rate constant of the reduction, which can be obtained by monitoring the decrease of the absorbance at $400 \mathrm{~nm}$ with time [25]. As Figure 5 shows, linear relationships of $\ln \left(A_{t} / A_{0}\right)$ versus reaction time indicate the reductions well match with
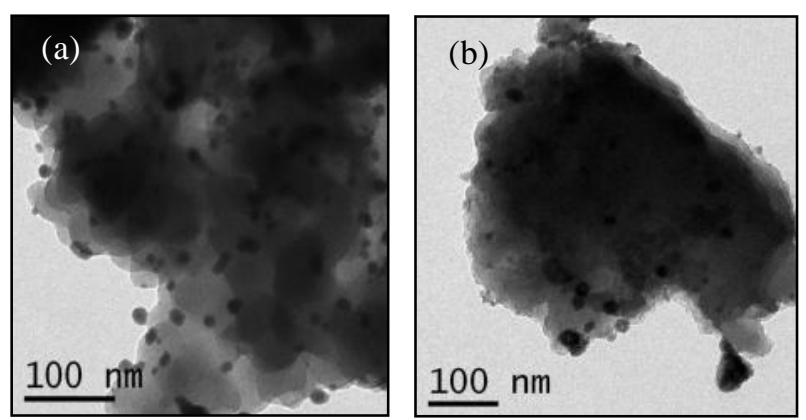

Figure 3. TEM images of (a) $3 \% \mathrm{Bi} / \mathrm{NC}$, and (b) $3 \% \mathrm{Bi} / \mathrm{AC}$

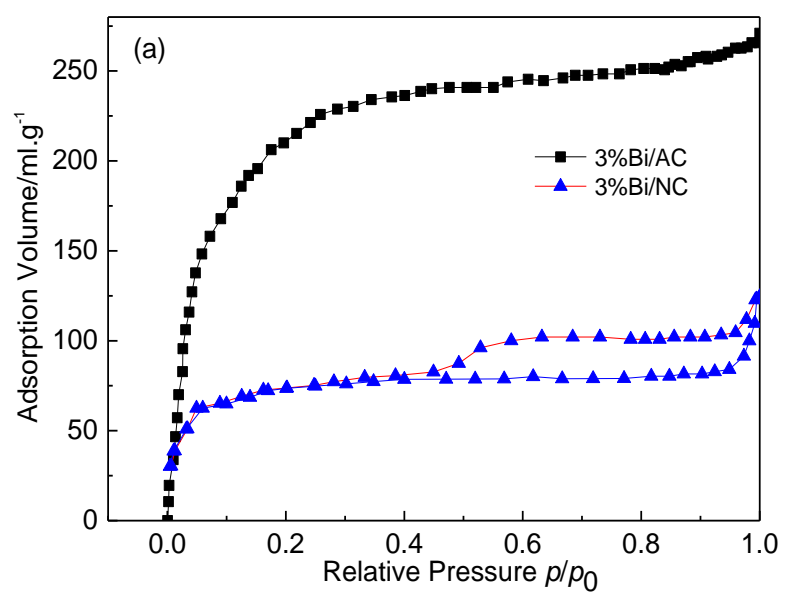

pseudo-first-order, but the line slopes are different for different catalysts. The $k$ calculated according to the line slope was $0.14,0.19,0.31$, and $0.38 \mathrm{~min}^{-1}$ for $1 \% \mathrm{Bi} / \mathrm{NC}, 3 \% \mathrm{Bi} / \mathrm{AC}$, $3 \% \mathrm{Bi} / \mathrm{NC}$, and $5 \% \mathrm{Bi} / \mathrm{NC}$, respectively. The results indicate that the catalytic activity increased with the $\mathrm{Bi}$ content in $\mathrm{Bi} / \mathrm{NC}$ catalyst. However, when the $\mathrm{Bi}$ content was the same, the activity of $3 \% \mathrm{Bi} / \mathrm{NC}$ catalyst was higher than that of $3 \% \mathrm{Bi} / \mathrm{AC}$ catalyst. Since the specific surface area of $3 \% \mathrm{Bi} / \mathrm{NC}$ is less than that of $3 \% \mathrm{Bi} / \mathrm{AC}$, it should be no contribution to the higher activity.

But the mesopores in $\mathrm{NC}$ can promote the transportation and reaction of reactant molecules. As shown in Figure 3, the Bi particles distribution in $\mathrm{NC}$ is more evenly than in $\mathrm{AC}$ due to the doped $\mathrm{N}$ atoms, so there are more active sites in $\mathrm{NC}$ than in $\mathrm{AC}$. The result further strengthens that nitrogen doping can significantly increase metal dispersion [8].

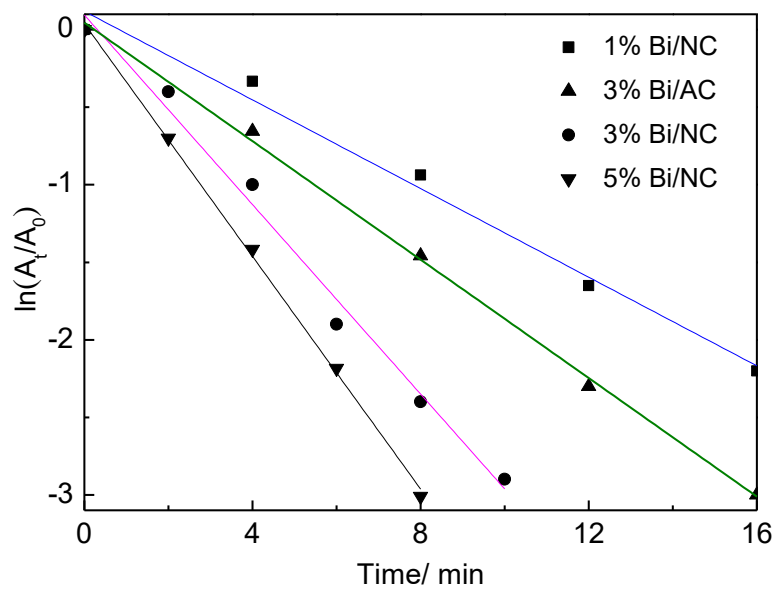

Figure 5. Activities of the catalysts

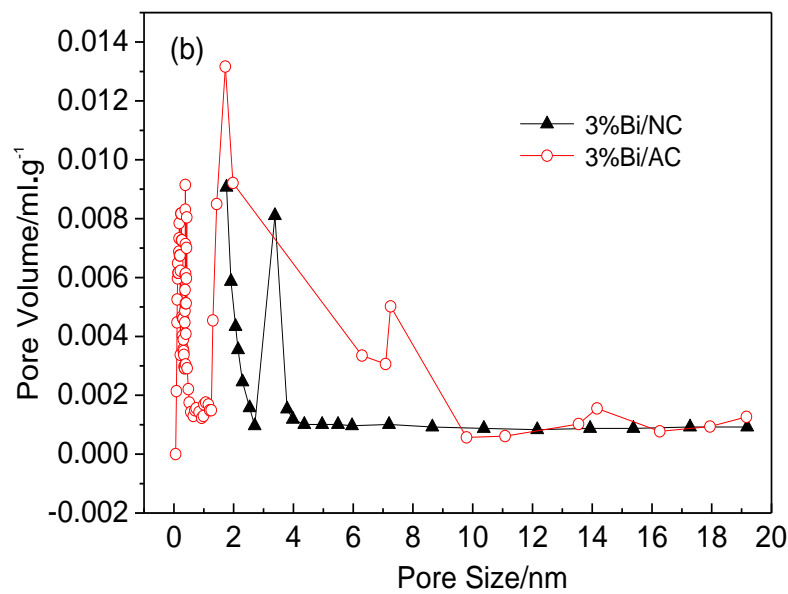

Figure 4. (a) Nitrogen adsorption-desorption isotherm and (b) pore size distribution of the catalysts 


\subsection{Reusability}

The reusability of catalyst is of importance for practical applications. To demonstrate the reusability of the catalysts, the as-prepared catalysts were recycled by centrifugation after the reaction, washed and recycled for the reduction. As shown in Figure 6, the activity of $3 \% \mathrm{Bi} / \mathrm{AC}$ decreased obviously after each use, the conversion of 4 -NP from $98.8 \%$ of the first cycle to $80.5 \%$ of the fourth cycle, but the activity of $\mathrm{Bi} / \mathrm{NC}$ was more stable because of strong attraction between $\mathrm{Bi}$ and $\mathrm{N}$ atoms [23]. Since the excessive active centers may aggregate and form other less active particles, the stability was also associated with $\mathrm{Bi}$ content in $\mathrm{NC}$, and the stability decreased with the $\mathrm{Bi}$ content increasing. The $1 \% \mathrm{Bi} / \mathrm{NC}, 3 \% \mathrm{Bi} / \mathrm{NC}$, and $5 \% \mathrm{Bi} / \mathrm{NC}$ can be used for $10,9,6$ cycles without obvious deactivation, respectively, and all of the conversions can still attained more than $97 \%$.

\section{Conclusions}

In summary, the $\mathrm{NC}$ was prepared by carbonization of MSG and $\mathrm{KOH}$, which was used as a carrier for $\mathrm{Bi} / \mathrm{NC}$ catalyst. The $\mathrm{Bi}$ particles distribute in the $\mathrm{NC}$ uniformly with diameter
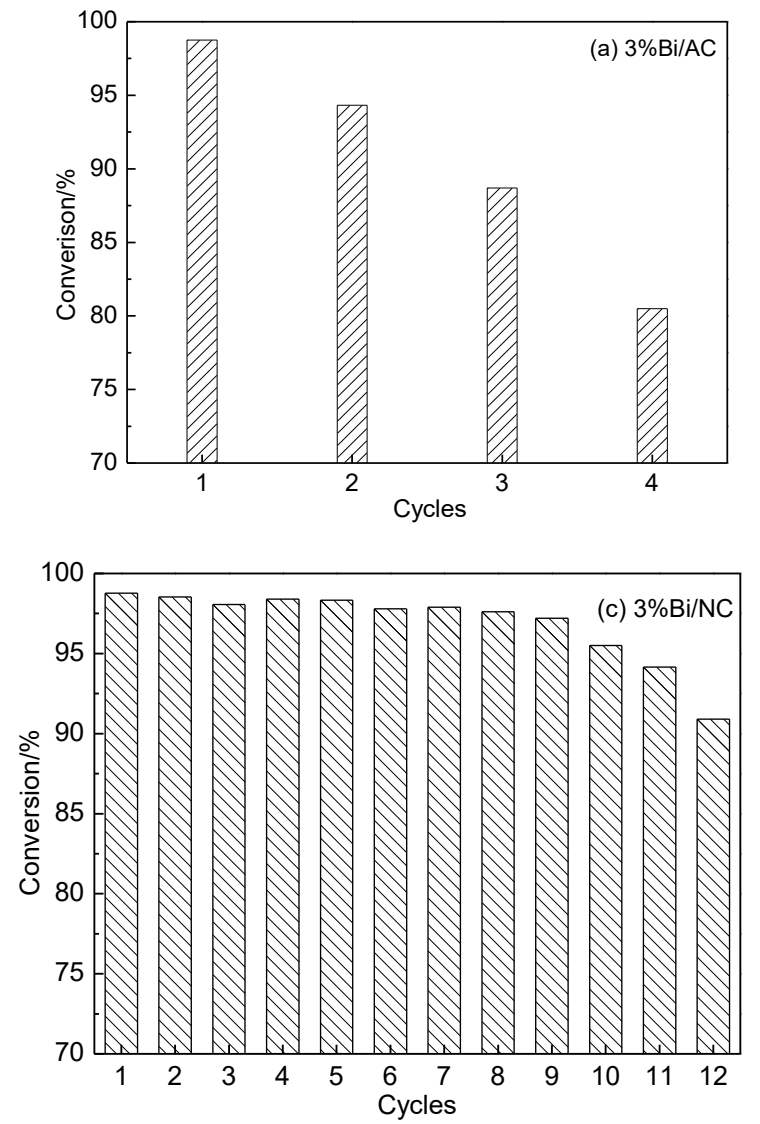

of $10-20 \mathrm{~nm}$ due to the chemisorption. The catalytic performances were investigated using the reduction of 4-NP with excessive $\mathrm{KBH}_{4}$ as a model reaction. The results indicate that the $\mathrm{Bi} / \mathrm{NC}$ catalysts have higher activity and better stability than the $\mathrm{Bi} / \mathrm{AC}$ catalyst. With the $\mathrm{Bi}$ content increasing, the activity of $\mathrm{Bi} / \mathrm{NC}$ increased, but the stability decreased.

\section{Acknowledgments}

The authors wish to acknowledge the financial supports from the Science and Technology Department of Jiangsu Province (BE2015041) and Jiangnan University (3516010241140190/ 002).

\section{References}

[1] Zhao, Z.K., Ge, G.F., Li, W.Z., Guo, X.W., Wang, G.R. (2016). Modulating the Microstructure and Surface Chemistry of Carbocatalysts for Oxidative and Direct Dehydrogenation: A Review, Chinese Journal of Catalysis. 37(5): 644-670.

[2] Kong, X.K., Chen, C.L., Chen, Q.W. (2014). Doped Graphene for Metal-free Catalysis. Chemical Society Reviews, 43(8): 2841-2857.
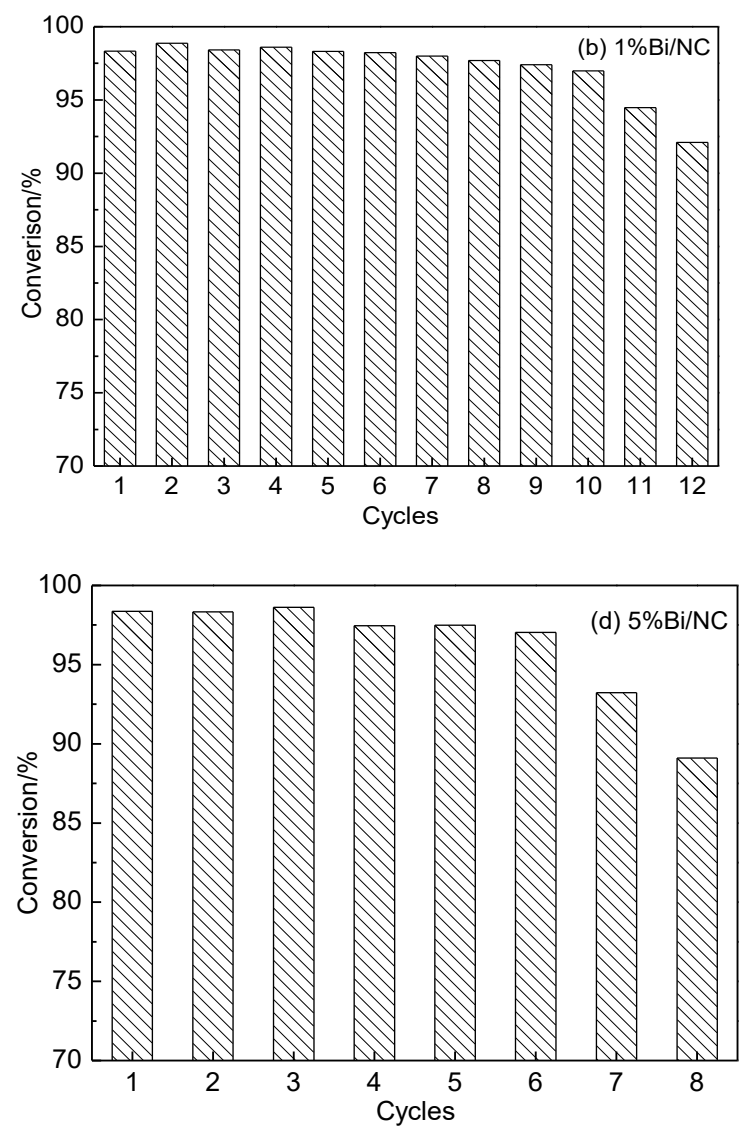

Figure 6. Reusability of the catalysts 
[3] Wang, H.B., Maiyalagan, T., Wang, X. (2012). Review on Recent Progress in Nitrogen-Doped Graphene: Synthesis, Characterization, and Its Potential Applications. ACS Catalysis, 2(5): 781-794.

[4] Yang, Y.F., Jia, L.T., Hou, B., Li, D.B., Wang, J.G., Sun, Y.H. (2014). The Effect of Nitrogen on the Autoreduction of Cobalt Nanoparticles Supported on Nitrogen-Doped Ordered Mesoporous Carbon for the Fischer-Tropsch Synthesis. Chemcatchem, 6(1): 319-327.

[5] Liu, J., Song, P., Ruan, M., Xu, W. (2016). Catalytic Properties of Graphitic and Pyridinic Nitrogen Doped on Carbon Black for Oxygen Reduction Reaction. Chinese Journal of Catalysis, 37(7): 1119-1126.

[6] Liang, Q.Y., Su, H., Yan, J., Leung, C., Cao, S.L., Yuan, D.S. (2014). N-Doped Mesoporous Carbon as a Bifunctional Material for Oxygen Reduction Reaction and Supercapacitors. Chinese Journal of Catalysis, 35(7): 1078-1083.

[7] Podyacheva, O.Y., Ismagilov, Z.R. (2015). Nitrogen-doped Carbon Nanomaterials: To the Mechanism of Growth, Electrical Conductivity and Application in Catalysis. Catalysis Today, 249(23): 12-22.

[8] Ombaka, L.M., Ndungu, P., Nyamori, V.O. (2013). Usage of Carbon Nanotubes as Platinum and Nickel Catalyst Support in Dehydrogenation Reactions. Catalysis Today, 217(5): 65-75.

[9] Hu, P., Meng, D.H., Ren, G.H., Yan, R.X., Peng, X.S. (2016). Nitrogen-Doped mesoporous carbon thin film for binder-free supercapacitor. Applied Materials Today, 5: 1-8.

[10] Wang, Z.Q., Sun, L.X, Xu, F., Zhou, H.Y., Peng, X.J., Sun, D.L., Wang, J.C., Du, Y. (2016). Nitrogen-Doped Porous Carbons with High Performance for Hydrogen Storage. Internal Journal of Hydrogen Energy, 41(20): 8489-8497.

[11] Kang, W.J., Li, H.B., Ai, M., Wei, S.Y., Gao, H.Z., Liu, J.F., Qian, Y.T. (2014). Synthesis of Nitrogen-Doped Carbon and Application in Highly Selective and Sensitive Dopamine Sensing. Materials Letters, 116(2): 374-377.

[12] Zhang, Z., Wang, G.C., Lai, Y.Q., Li, J., Zhang, Z.Y., Chen, W. (2015). Nitrogen-Doped Porous Hollow Carbon Sphere-Decorated Separators for Advanced Lithiume-Sulfur Batteries. Journal of Power Sources, 300: 157163.

[13] Niu, W.H., Li, L.G., Chen, S.W. (2017). Recent Progress in Template-Assisted Synthesis of Nitrogen-Doped Porous Carbon for Oxygen Electroreduction. Journal of Electrochemistry, 23(2): 110-122.
[14] Qian, W.J., Zhu, J.Y., Zhang, Y., Wu, X., Yan, F. (2015). Condiment-Derived 3D Architecture Porous Carbon for Electrochemical Supercapacitors. Small, 11(37): 4959-4969.

[15] Wang, Z.Z., Zhai, S.R., Zhai, B., Xiao, Z.Y., An, Q.D. (2014). Preparation and Catalytic Properties of Nano-Au Catalytic Materials Based on the Reduction of 4-Nitrophenol. Progress in Chemistry, 26(2/3): 234-247.

[16] Noh, J-H., Meijboom, R. (2014). Catalytic Evaluation of Dendrimer-Templated $\mathrm{Pd}$ Nanoparticles in the Reduction of 4Nitrophenol Using Langmuir-Hinshelwood Kinetics. Applied Surface Science, 320: 400413.

[17] Guo, P.C., Tang, L., Zeng, G.M., Huang, B.B., Dong, H.R., Zhang, Y., Zhou, Y.Y., Deng, Y.C., Ma, L.L., Tan, S.R. (2016). Catalytic Reduction-Adsorption for Removal of $p$ Nnitrophenol and Its Conversion $p$ Aminophenol from Water by Gold Nanoparticles Supported on Oxidized Mesoporous Carbon. Journal of Colloid and Interface Science, 469: 78-85.

[18] Li, Y.Z., Cao, Y.L., Xie, J., Jia, D.Z., Qin H.Y., Liang, Z.T. (2015). Facile Solid-State Synthesis of Ag/Graphene Oxide Nanocomposites as Highly Active and Stable Catalyst for the Reduction of 4-Nitrophenol. Catalysis Communications, 58: 21-25.

[19] Salvador, J.A.R., Pinto, R.M.A., Silverstre, S.M. (2009). Recent Advances of Bismuth (III) Salts in Organic Chemistry: Application to the Synthesis of Heterocycles of Pharmaceutical Interest. Current Organic Synthesis, 6: 426-470.

[20] Qiu, Y.Y., Yang, C.Q., Huo, J., Liu, Z.G. (2016). Synthesis of Co-N-C Immobilized on Carbon Nanotubes for Ethylbenzene Oxidation. Journal of Molecular Catalysis A: Chemical, 424: 276-282.

[21] Jiang, Y.Q., Chowdhury, S., Balasubramanian, R. (2017). Nitrogen-Doped Graphene Hydrogels as Potential Adsorbents and Photocatalysts for Environmental Remediation. Chemical Engineering Journal, 327: 751-763.

[22] Ma, D.C, Zhao, J.Z., Chu, R., Yang, S.S., Zhao, Y., Hao, X.L., Li, L.Z., Zhang, L., Lu, Y., Yu, C.Z. (2013). Novel Synthesis and Characterization of Bismuth Nano/Microcrystals with Sodium Hypophosphite as Reductant. Advanced Powder Technology, 24(1): 79-85.

[23] Zhao, W.L., Zhu, M.Y., Dai, B. (2017). CobaltNitrogen-Activated Carbon as Catalyst in Acetylene Hydrochlorination. Catalysis Communications, 98: 22-25. 
[24] Du, X.Y., He, J., Zhu, J., Sun, L.J., An, S.S. (2012). Ag-Deposited Silica-Coated $\mathrm{Fe}_{3} \mathrm{O}_{4}$ Magnetic Nanoparticles Catalyzed Reduction of $p$-Nitrophenol. Applied Surface Science, 258(7): 2717-2723.
[25] Gao, J., Jian, X., Wen, S.X., Hu, J., Liu, H.L. (2015). Plasma-Assisted Synthesis of Ag Nanoparticles Immobilized in Mesoporous Cellular Foams and Their Catalytic Properties for 4-Nitrophenol Reduction. Microporous and Mesoporous Materials, 207: 149-155. 